\title{
A System for Segmentation and Bi-Level Cell Tracking
}

\author{
R. Guzmán Cabrera, J. R. Guzmán Sepúlveda, J. C. Barrón-Amieva, J. A. Gordillo Sosa, A. González \\ Parada, M. Torres Cisneros, G. Aviña-Cervantes, and O. G. Ibarra-Manzano
}

\begin{abstract}
Measurement of the proliferative behaviors of in vitro cells is important to many biomedical applications ranging from basic biological research to advanced applications, such as drug synthesis, stem cell manufacturing, and tissue engineering. The detection of borders within an image constitutes a process of digitalization of the image. Once the digitized image is obtained, the next step is the application of a specific process consisting in applying algorithms that allow the obtaining of raw data of the image. In this case, the applied algorithm to the digitized images was the Canny algorithm. This work presents a system to compute a vector representation for a selected cell of an image. The representation is in bi-level raster image.
\end{abstract}

Index Terms-Bi-level, cell tracking, raster image.

\section{INTRODUCTION}

The basic study of human form and function has underpinned the establishment of modern medicine, and is well recognized as having evolved in large part from the original detailed topographic drawings of human anatomy by Leonardo da Vinci, who firmly believed in the power of imaging and visualization as a critical tool for providing key insights into nature and human health. A more recent iteration of that same fundamental concept is evidenced by the National Library of Medicine's 'The Visible Human Project ${ }^{\circledR}$ ', in which the combination of a number of powerful clinical imaging techniques with modern computing methods resulted in complete atlases of the human body at unprecedented resolution.

Computational image analysis tools for semi-automated and automated tracking of single cells or cell clusters within live biological systems have been developed and reported since early 1980s. In order to produce quantitative and statistically relevant results, large amounts of data are required (say, several sequences, each containing several hundredth of images), and automatic image analysis algorithms become necessary. One frequent aim is to extract

Manuscript received June 13, 2012; revised July 22, 2012.

R. Guzmán-Cabrera, M. Torres Cisneros, G. Aviña-Cervantes, and O. G. Ibarra-Manzano are with the Grupo de NanoBioFotónica, DICIS, Universidad de Guanajuato; Salamanca, Gto., México (e-mail: guzmanc@ugto.mx, mtorres@ugto.mx, avina@ugto.mx, ibarrao@ugto.mx).

J. R. Guzmán-Sepulveda is with the Departamento de Electrónica, UAM Reynosa-Rodhe, Universidad Autónoma de Tamaulipas. Carr. Reynosa-San Fernando S/N, Reynosa, Tamaulipas 88779, México (e-mail: jrafael_guzmans@yahoo.com.mx)

J. C. Barron-Amieva is with the Centro Universitario de Negocios. Blvd las Reynas \#100, Colonia las Reynas, Salamanca, Gto., Mexico (e-mail: jbarron.amieva@live.com.mx)

J. A. Gordillo Sosa is with the Universidad Tecnológica del Suroeste de Guanajuato. Carretera Valle-Huanimaro Km. 1.2, Valle de Santiago, Gto. México.

A. González Parada is with the DICIS, Universidad de Guanajuato; Salamanca, Gto., México (e-mail: gonzaleza@ugto.mx) from the image sequence the complete description of cell positions, shape, and motion across time, leading, in the case of dividing cells, to a space-time lineage. These segmentation and dividing/tracking issues have to be solved in the most reliable manner, since human post-processing is the limiting factor of the rate of processed data.

Currently the most important imaging tool for studying dynamic processes in living cells is still light microscopy. Biological research is impossible to imagine without a microscope. Latest generations of microscopes, able to produce huge arrays of multidimensional data, only distantly resemble Leeuwenhoek's first microscope. Every advance in visualization techniques and hardware brings us one step closer to understanding life, e.g., how genome information gives identity to cells, how cells constitute organisms and how errant cells cause disease. The use of light microscopes for biological experimentation and investigation goes back to Antoni van Leeuwenhoek (1632-1723), who discovered the motion of bacteria, sperm cells, blood cells and more, using a simple magnifying lens. It is only since relatively recently, however, that light microscopy became mature enough to allow in vivo imaging of molecular complexes and even single molecules. Apart from substantial improvements in optics hardware and the development of increasingly sensitive electronic imaging sensors, a key factor was the discovery, cloning, and expression of the jellyfish green fluorescent protein (GFP).

This enabled visible fluorescence to be encoded into a specific gene of interest, which, in turn, enables one to tag and optically detect virtually any protein of interest in living cells. In recent years, many GFP variants have been developed with different spectral properties, enabling simultaneous detection of differently labeled proteins and studying their interaction. Since the first images of cultured mammalian cells recorded to film using an microscope were published in the United States in 1945, the ultrastructural analysis of mammalian cells/tissue has relied almost entirely on extrapolating the three-dimensionality of the structures/compartments under investigation from sets of two-dimensional (2D) images.

A few examples of biological molecular dynamic processes and images acquired for studies into these phenomena may help one to appreciate the challenges of tracking. One of the intracellular structures being investigated intensively in biology is the cytoskeleton, which consists of several subsystems of densely interwoven and highly plastic networks of filamentous polymers. One category of cytoskeleton polymers is constituted by the microtubules. These are required for a variety of cellular functions and their dynamic behavior is regulated by many factors. 
Understanding physiological processes in health and disease and developing adequate drugs requires the imaging and analysis of the (morpho) dynamic behavior of single cells or cell clusters in tissues under normal and perturbed conditions. This typically involves the tracking and quantification of large numbers of cells in time-lapse fluorescence, phase-contrast, or intravital microscopy data sets consisting of hundreds to many thousands of image frames, making manual analysis no option, especially in 3D. The automation of these tasks faces several challenges, including the generally poor image quality (low contrast and high noise levels), the varying density of cell populations due to division, cells entering or leaving the field of view, and the possibility of cells touching each other without showing sufficient image contrast. Many computerized methods for cell tracking have already been proposed, and some of these have found their way to commercial and open-source software tools, but the consensus arising from the literature seems to be that any specific tracking task requires dedicated (combinations of) algorithms to obtain optimal results. Nevertheless, several trends can be observed in the development of new cell tracking methods, suggesting the superiority of particular algorithms.

The study of cell movement is fundamental to research in embryological development, wound healing, immune defense, tumor cell invasion, and metastasis [1]. Cell migration is conventionally monitored by using time-lapse microscopy in combination with different 2-D or 3-D tissue substrata. For the detection of changes in cell shape and/or relative position with time from serial images a precise and robust segmentation procedure is required to discriminate between the cell boundary and the surrounding tissue.

Since the apparition of the first proposals of computational image analysis tools for semi-automated and automated tracking of single cells or cell clusters within live biological systems in the early 1980s, a growing interest on cell dynamics has arisen due to the possibility of studying several aspects of the cellular processes, such as stem/parent identification for lineage determination and their behavior in the face of alternative cell fates, cell aging, response to different culture conditions, characterization of proliferation, social interactions during biological processes such as wound healing, and the obtainment of precise information about cell behavior during general biological processes such as migration, mitosis, apoptosis, shape deformation of individual cells, and their interactions among cells [1-3].

In order to produce quantitative and statistically relevant information, large amounts of data are required (i.e. several sequences, each one containing several hundreds of images), and automatic image analysis algorithms become necessary. Typical experiments produce over 100 gigabytes (GB) of image data consisting of about 40,000 frames, representing thousands of cells in each frame. This makes automated tracking and cell analysis critical to efficiently study the underlying biological mechanisms. However, the high processing demand, the varying density of the cell culture (i.e. cells dividing/dying, leaving/re-entering the field-of-view), and the complexity of the cellular topologies (i.e. shape deformation, close contact, and partial overlap) impose many challenges to the existing tracking techniques.
There are numerous studies reported in the literature in which vast amounts of image data are generated and where the dynamics of hundreds or thousands of particles need to be quantitatively analyzed in order to reveal detailed information related to the functional behavior thus allowing detect rare events suggestive of functional heterogeneity. This may lead to the formulation of comprehensive models of molecular processes. It is evident that manual tracking is an intensive labor which usually results in costly, inaccurate, and poorly reproducible extracted information, furthermore, due to manual processing is time consuming only a small fraction of the data can be analyzed in this manner. This calls for renewed efforts in developing computational image analysis tools.

Several approaches using a variety of techniques have been previously reported. In general, the proposed tracking methods agree with the rough classification found in [4], in which it is settled that there are two general ways to perform cell tracking: the first one finds the object contour in the current frame given an initial contour from the previous frame; the second one segments the cell regions in two or more frames simultaneously and then resolves the association functions between the regions of interest.

Despite very good results can be found in the literature in terms of geometrical and topological treatments, both tracking techniques still present some problems when they deal with dividing cells, cells entering/leaving the field of view, cells merging into a cluster, and cells touching or overlapping [4-9]. In this regard, several efforts have been proposed to handle the problems mentioned above, among which are parent/stem association by using the local association method [10], adaptive thresholding method [11], data association by using the graph theoretic minimum cost flow framework considering various realistic cell behaviors (i.e. migration, mitosis, overlap, entering and leaving) [12], incorporation of a Markov random field prior distribution into the particle filter framework [13,14], split/merged measurements assuming that the number of objects is fixed [15], and contour tracking based on partial contour matching [2].

In this work, we will focus on the extraction and vectorization of individual lines that may have arbitrary width, rough contours, crossings, and branches.

The proposed algorithm can be classified as a model-based contour evolution since it performs the cell tracking by successively filtering the image in such way that the information obtained from the contour detection from a previous frame is used to detect the contour in the current frame but taking certain positions of specific pixels as reference for the following processing. The Canny algorithm was used as filter for the contour detection. By storing the coordinates of the positions of interest and using the information obtained from the contour detection, it is possible to predict the cell trajectory based on the analysis of a few frames.

\section{MeTHODOLOGY}

The contour detection algorithm used in our method is based on that described in [9].This algorithm allows 
detecting the entire outline of an arbitrary 8-shaped object in the counter clockwise direction. The outline will be an 8 -connected set of foreground pixels, each of them having one or more neighbors belonging to the background. The general scheme of the method proposed in this work is depicted in Fig. 1.

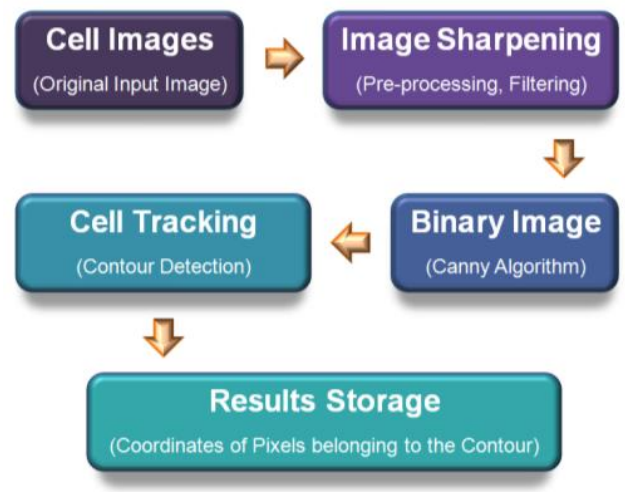

Fig. 1. General scheme of the proposed canny-based algorithm for cell tracking.

Given an initial starting pixel $\mathrm{S}_{\mathrm{i}}$, the next outline pixel and next starting pixel are chosen as follows: search the neighbors in the counter clockwise direction, starting with $\mathrm{S}_{\mathrm{i}}$, until a transition from background to foreground is found. The first foreground pixel thus found will be the next outline pixel and the last background pixel will be the next initial pixel, respectively. This process will continue with every transition found.

The general scheme of the computational approach proposed by Canny is shown in Fig. 2. The Canny algorithm can be summarized in five main stages: smoothing, finding the intensity gradients, non-maximum suppression, double thresholding, and edge tracking [16].

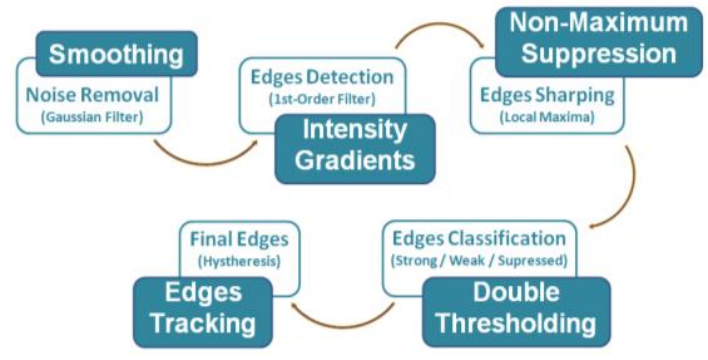

Fig. 2. General diagram of the canny algorithm.

Raw unprocessed image data are always susceptible to present intrinsic noise due to the mechanism through which the image was constructed/taken (i.e. camera, X-ray, microscopy). The smoothing stage has the purpose of removing noise from the original image in order to prevent the detection of mistaken edges in further stages and then reduce the probability of constructing false contours for tracking. Smoothing is performed by convolving the image with a two-dimensional Gaussian filter, which is typically symmetric and centered at the central pixel of the mask thus provoking that the resulting image, depends only on the standard deviation $\sigma$. The resulting image is a slightly blurred version of the original which due to the noise compression, which is not significantly affected by a single noisy pixel.

In the next stage, finding the intensity gradients allows finding the edges in the image. Since an edge can be oriented in a variety of directions, Canny algorithm performs edge detection by applying four filters in order to detect horizontal, vertical, and diagonal edges. Canny algorithm approximates the intensity gradients in the image by using first-order filtering masks, such as Sobel or Roberts kernels. The effect of increasing $\sigma$ in the noise-removal stage is the gradients are much smoother, which means that $\sigma$ determines the sharpness of the detected edges in the current stage. Once the edges have been detected, the next stage consists on sharpening them. The main purpose of this step is to convert the rough (blurred) edges resulting from the image of the gradient magnitudes to fine (sharp) edges. This step distinguishes the Canny algorithm from other algorithms since it takes into account the neighbors of each edge along the direction of the local gradient. This step is performed by preserving all local maxima in the gradient image and deleting everything else. Furthermore, the gray-intensity value assigned to the sharp edges is directly related to the strength of the original edges along the gradient direction.

Despite it is highly probable than the sharp edges remaining in the image after the non-maximum suppression are the true edges of the images, the gray-intensity values of these edges are accented by the pixel-by-pixel strength thus causing that some edges may be formed by underlying noise or color variations (typical for rough surfaces). In order to discriminate between true/false edges, a threshold is used as indicator of the permanency of the edges. The Canny algorithm uses a double thresholding (i.e. high and low thresholds) which leads to a classification of the edges with three possible scenarios: strong edges, weak edges, and suppressed edges.

In this regard, edge pixels with intensity values greater than the high threshold are marked as strong; edge pixels with intensity values less than the high threshold but greater than the low threshold are marked as weak; and finally, edge pixels with intensity values less than the low threshold are suppressed and are not considered for the resulting image.

Finally, the last stage of the Canny algorithm determines which edges will appear in the output image by a simple criterion: strong edges are immediately included while weak edges are included only if they are connected to a strong edge, otherwise, they are ignored. Once this stage is complete, a binary image containing the edges of the input image is obtained as the output image of the Canny algorithm.

Despite it is possible that high noise or large color variations result in a strong edge, this condition is almost never achieved. By selecting adequate values for the standard deviation and both thresholds, noise and color variations will only result in weak edges. Furthermore, color variations usually generate weak edges uniformly distributed on the entire image independently of the true edges [16]. The previous fact results in a low probability to find weak edges connected to strong edges thus allowing that the edges include in the output image are only due to the true edges of the input image.

\section{TEST AND RESUlts}

Since the appearance and behavior of cells can be quite different from particles, the image processing techniques developed to track them are usually also quite different. In 
either case, there are generally two sides to the tracking problem: 1) the recognition of relevant objects and their separation from the background in every frame (the segmentation step), and 2) the association of segmented objects from frame to frame and making connections (the linking step).

Fig. 3 depicts the original image, which consists of a set of cells. The pictures were provided by [9].

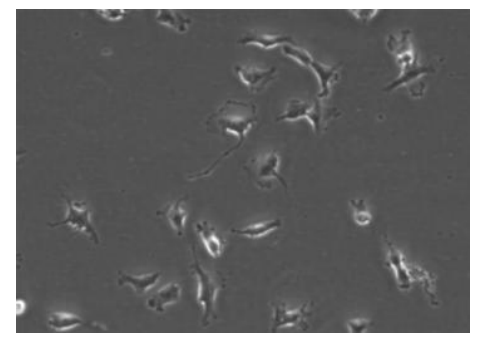

Fig. 3. Original image.

The read image is converted to a gray-scale image in order to apply the edge detection over one single dimension. As mentioned, Canny filter is used as base of the image filtering due to its capability of being adjusted for reducing noise in the resulting image.

Cell tracking methods generally consist of two main image processing steps: 1) cell segmentation (the spatial aspect of tracking), and 2) cell association (the temporal aspect). Segmentation process consists on dividing an image into (biologically) meaningful parts (segments), resulting in a new image containing for each pixel a label indicating to which segment it belongs. It basically serves to the discrimination between "foreground" versus "background".

Fig. 4 shows the filtered image after applying the Canny filter. The main objective is to sample the gray level at various places near the cells in the picture and estimate the local gray-level thresholds that can be used to define seeds (low threshold) and extra-cell space (high threshold).

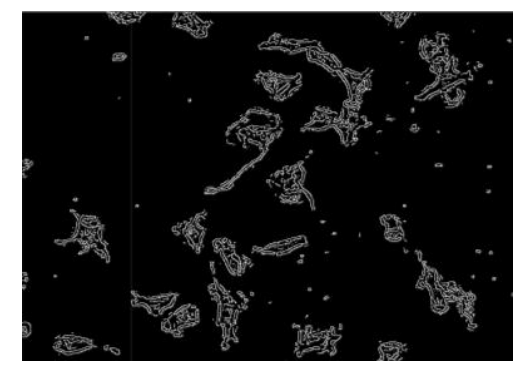

Fig. 4. Filtered image, is using a filter canny.

Once a first contour detection has been performed, the next step consists on tracking the neighboring selected pixel in the counter clockwise direction, this way the pixel that matches the intensity value of the pixel edge will be stored as part of the outline shape. Then the last pixel identified as part of the contour will be taken as pivot for the next filtering and the searching process repeats using the next neighboring pixel until the full contour has been detected.

Now that we have an over- and under-segmentation, we can generate all potential cells that are consistent with these bounds - in other words, all the unions of blobs on "linear subgraphs" of the connection graph described in the previous section. Once the cells are properly segmented in all frames of a time-lapse image sequence, and the correct associations have been established between successive frames, it is relatively easy to compute (morpho) dynamic features that may reveal biologically relevant phenomena. For example, variabilities in cell shape (within populations or per cell) over time can be very effectively studied using statistical tools such as principal component analysis of the cell outlines.

In images where the cells have sufficiently and consistently different intensities than their surroundings, they are most easily segmented by thresholding, which labels pixels above the intensity threshold as "object" and the remainder as "background", after which disconnected regions can be automatically labeled as different cells. In the case of severe noise, auto fluorescence, photo bleaching (in fluorescence microscopy), poor contrast, gradients, or halo artifacts (in phase-contrast or differential interference contrast microscopy), thresholding will fail, and more sophisticated segmentation approaches are needed.

The simplest approach to solving the subsequent association problem is to link every segmented cell in any given frame to the nearest cell in the next frame, where "nearest" may refer to spatial distance but also to difference in intensity, volume, orientation, and other features. This nearest-neighbor solution works well as long as the cells are well separated in at least one of the dimensions of the feature space. Essentially, this criterion also applies to so-called online cell tracking approaches, which alternate between segmentation and linking on a per-frame basis. For instance, template matching, mean-shift processing, or deformable model fitting is applied to one frame, and the found positions or contours are used to initialize the segmentation process in the next frame, and so on, which implicitly solves the linking problem (see Fig. 5).

Fig. 5 shows the filtered image with the edges marked. This is achieved by selecting the cell of interest. However, the basic concepts underlying the vast majority of published methods are virtually the same. The commonly used approach in motion tracking consists of at least the following steps: preprocessing the image data, detecting individual particles every time step, linking particles detected at successive time points, and analyzing the results.

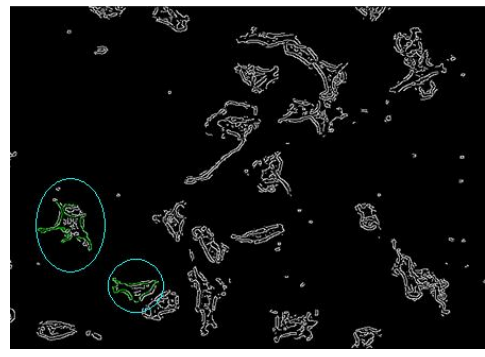

Fig. 5. Filtered image with edges marked.

Assuming the image as a 2D space, the coordinates of the pixels belonging to the contour will be stored as ordered pairs. By storing the coordinates of the positions of interest and using the information obtained from the contour detection, such as relevant issues about geometry and topology, it is possible to build a probabilistic model that allows predicting the cell trajectory based on the analysis of only a few frames. The proposal of building a mathematical model that describes 
the motion of the cells in the image not only helps to decrease the computational costs but offers an alternative to face the problem of overlapping and/or touching cells, entering/leaving cells, dividing cells, and cells merging into a cluster.

\section{CONCLUSION}

The breadth of medicine that stands to benefit from improved knowledge of cell-based therapies is vast. In vivo cell tracking offers insight into the underlying biological processes of new cell-based therapies, with the aim to depict cell function, migration, homing and engraftment at organ, tissue, cellular and molecular levels. Molecular imaging has expanded the possibilities through different imaging modalities and contrast agents of which this article will focus upon optical imaging.

Until recently, it is possible to manually identify incidents of mitosis because mitotic cells tend to retract, round up, and exhibit intensified surrounding halos under phase contrast illumination for short-period, small-scale studies. The proposed method does not depend on empirical parameters or ad hoc image pre-processing, which makes it suitable, robust, and straightforward for adapting to different types of cells. Since it is also a simple algorithm, based on repetitive contour detection taking specific positions from the contour identification of previous frames, it allows cell tracking with relatively low computational costs. Furthermore, the proposed algorithm can be easily incorporated to a more complex system in which a mathematical model can be build to describe the motion of the cells in the image by analyzing only a few frames thus providing an alternative to face overlapping and/or touching cells, entering/leaving cells, dividing cells, and cells merging into a cluster since the trajectory of the cells can be approximated and then cells can be discriminated and differently treated for these special cases.

\section{FUTURE WORK}

There is now a clear desire/need within the international biology community, the presence of diseases such as diabetes and cancer, open up important research lines for biomedical research communities to understand the 'bigger picture' with respect to the cell as complex systems, and using a precise spatial framework as a 'foundation' is considered by many in systems biology as a fundamental prerequisite to developing a genuine capacity to accurately model changes in the spatio-temporal coordinates of complex cellular events in silico and ultimately, for predicting the pathophysiology of chronic diseases like diabetes and cancer in the future.

By combining new mathematical and computational tools for precisely extracting useful, novel and quantitative insights into $2 \mathrm{D}$ and $3 \mathrm{D}$ cellular organization from these enormous datasets, we foresee a unique opportunity to begin mapping structural phenotypes for cell function versus dysfunction onto complete sets of $2 \mathrm{D}$ and $3 \mathrm{D}$ spatial coordinates for cell organization with nanometer precision.

A future area of focus that would improve the sensitivity of cell tracking and possibly quantification with optical imaging would be the generation of molecular markers that could identify engrafted cells and the nature of their progeny.

\section{REFERENCES}

[1] E. J. Stewart, R. Madden, G. Payl, and F. Taddei, "Aging and death in an organism that reproduces by morphologically symmetric division," PloS Biol vol. 3, no. 2, pp. 45, 2005.

[2] R. Bise, K. Li, S. Eom, and T. Kanade, "Reliably tracking partially overlapping neural stem cells in dic microscopy image sequences," MICCAI Workshop on Optical Tissue Image Analysis in Microscopy Histopathology and Endoscopy, vol. 12, pp. 67-77, 2009.

[3] A. J. Hand, T. Sun, D. C. Barber, D. R. Hose, and S. Macneil, "Automated tracking of migrating cells in phase-contrast video microscopy sequences using image registration," Journal of Microscopy, vol. 234, Pt. 1 2009, pp. 62-79, 2009.

[4] R. Bise, Z. Z. Yin, and T. Kanade, "Reliable cell tracking by global data association," IEEE International Symposium on Biomedical Imaging , 2011.

[5] T. Kanade, Z. Z. Yin, R. Bise, S. Huh, S. Eom, M. F. Sandbothe, and M. Chen, "Cell image analysis: algorithms, system and applications," IEEE Workshop on Applications of Computer Vision (WACV), pp. 374 $-381,2011$.

[6] A. Liu, K. Li and T. Hao, "A hierarchical framework for mitosis detection in time-lapse phase contrast microscopy image sequences of stem cell populations," in Book Medical Imaging, Ed. Okechukwu Felix Erondu, 2011.

[7] S. Eom, S. Huh, D. F. E. Ker, R. Bise, and T. Kanade, "Tracking of hematopoietic stem cells in microscopy images for lineage determination," Journal of Latex Class Files, vol. 6, no. 1, pp. 1 - 9 , 2007.

[8] K. Li, E. Miller, L. Weiss, P. Campbell, and T. Kanade, "Online tracking of migrating and proliferating cells imaged with phase contrast microscopy", Proceedings of the Conference on Computer Vision and Pattern Recognition Workshop (CVPRW'06), pp. 65 - 72, 2006.

[9] O. Debeir, P. V. Ham, R. Kiss, and C. Decaestecker, "Tracking of migrating cells under phase-contrast video microscopy with combined mean-shift processes," IEEE Transactions on Medical Imaging, vol. 4 , no. 6, pp. 697-71, 2005.

[10] K. Li, E. D. Miller, M. Chen, T. Kanade, L. E. Weiss, and P. G. Campbell, "Cell population tracking and lineage construction with spatiotemporal context," Medical Image Analysis, vol. 12, no. 5, pp. 546-566, 2008.

[11] O. Al-Kofahi, R. Radke, S. Goderie, Q. Shen, S. Temple, and B. Roysam, "Automated cell lineage construction: A rapid method to analyze clonal development established with murine neural progenitor cells," Cell Cycle, vol. 5, no. 3, pp. 327-335, 2006.

[12] D. Padfield, J. Rittscher, and B. Roysam, "Coupled minimum-cost flow cell tracking," Information Processing in Medical Imaging, pp. 374-385, 2009.

[13] Z. Khan, T. Balch, and F. Dellaert, "An MCMC-based particle filter for tracking multiple interacting targets," European Conference on Computer and Vision, vol. IV, pp. 279-290, 2004.

[14] N. J. Gordon, D. J. Salmond, and A. F. M. Smith, "Novel approach to nonlinear/non-gaussian bayesian state estimation," IEEE Proceedings- $F$, vol. 140, no. 2, pp. 107-113, 1993.

[15] Z. Khan, T. Balch, and F. Dellaert, "Multitarget tracking with split and merged measurements," IEEE Computer Society Conference on Computer, Vision and Pattern Recognition, pp. 605-610, 2005.

[16] J. Canny, "A computational approach to edge detection," IEEE Transactions on Pattern Analysis and Machine Intelligence, PAMI-vol. 8, no. 6, pp. $679-698,1986$.

Rafael Guzmán-Cabrera is a full time Professor, Faculty of Engineering Mechanics Electrics and Electronics, Guanajuato University, $\mathrm{PhD}$ in Pattern Recognition and Artificial Intelligence from Polytechnic University of Valencia, Spain. Contributor in research projects in the area of electrical engineering, Pattern Recognition and Artificial Intelligence

José R. Guzmán-Sepúlveda is a student of MS degree in Electrical Engineering at the Multidisciplinary Faculty Reynosa-Rodhe in Tamaulipas, Mexico. Contributor in national research projects in the area of Electrical Engineering related to Pattern Recognition and Artificial Intelligence (University of Guanajuato). Contributor in research projects abroad in the area of Optics and Photonics related to the design, fabrication, characterization, and testing of photonics sensors (UCF-CREOL). 